\title{
Force irradiation effects during upper limb diagonal exercises on contralateral muscle activation
}

\author{
Rosa Abreu, Alfredo Alexandre Lopes, Andreia S.P. Sousa, Soraia Pereira, Marcelo P. Castro \\ Department of Physiotherapy, School of Allied Health Sciences of Porto (ESTSP), Polytechnic Institute of Porto, Portugal
}

A B S T R A C T

\begin{abstract}
The aim of this study was to explore the force irradiation effects of upper limb isometric diagonal exercises on shoulder muscle activities. Interactions among diagonal directions, contraction intensities (moderate and maximum) and sex were assessed. Thirty healthy subjects ( 11 males) performed isometric unilateral diagonal exercises based on proprioceptive neuromuscular facilitation technique in an isokinetic dynamometer with their dominant upper limbs. The second diagonal for flexion and for extension were assessed while the participants performed their maximum isometric torque (MIT) and at $25 \%$ of their MIT. During the exercise the muscle activity of the medial deltoid, pectoralis major and upper trapezius in the non-dominant (non-exercised) upper limbs of the participants was recorded by surface electromyography. The highest muscle activity occurred in the upper trapezius during the diagonal for flexion (27\% of maximum isometric voluntary contractions). Upper trapezius and pectoralis major were more active during the diagonal for flexion than diagonal for extension $(p<0.001)$, while similar values between both diagonals were observed for the medial deltoid $(p>0.05)$. In conclusion, we observed that force irradiation during upper limb diagonal exercises is affected by diagonal direction, contraction intensity and sex when performed by healthy participants.
\end{abstract}

\author{
Keywords: \\ Contralateral muscle activity \\ Cross education \\ Force irradiation \\ Proprioceptive neuromuscular facilitation \\ Unilateral resistance
}

\section{Introduction}

Proprioceptive neuromuscular facilitation (PNF) is a concept of treatment widely used in rehabilitation to improve the performance of the neuromusculoskeletal system through the stimulation of muscle and joint proprioceptors (Gontijo et al., 2012; Kofotolis and Kellis, 2007; Meningroni et al., 2009). This approach uses either dynamic contractions associated with stretching or isometric contractions (Hazaki et al., 1996; Kofotolis and Kellis, 2007; Voss et al., 1985). During both types of contractions maximal resistance is commonly applied, although moderate contractions can also be used (Voss et al., 1985). The hallmark of the PNF technique is the use of diagonals or spiral movements (Adler et al., 2000; Hazaki et al., 1996; Meningroni et al., 2009; Sato and Maruyama, 2009). One principle of PNF is the force irradiation, which is based on fact that the stimulation of strong and preserved muscles produces activation of the contralateral injured and weak muscles
(Pink, 1981; Voss et al., 1985). Neuromuscular mechanisms, such as proprioception of muscle spindles and Golgi tendon organ, and joint receptors are behind PNF force irradiation. The strength gains of the contralateral untrained homologous muscles are referred to as cross education or cross training (Carroll et al., 2006; Munn et al., 2004). Although the increase of magnitude of muscle activity during cross education is controversial, substantial levels of contraction in the contralateral untrained muscles have been observed (Devine et al., 1981; Dimitrijevic et al., 1992; Moore, 1975; Panzer et al., 2011; Pink, 1981; Røe et al., 2000). The force irradiation is likely to be one of the mechanisms underlying the cross education phenomenon (Devine et al., 1981; Dimitrijevic et al., 1992; Moore, 1975; Panzer et al., 2011; Pink, 1981).

The force irradiation effect depends on the abundance of stimulus from the central motor pathways for the muscles in contraction and also of the afferent feedback to the contralateral motor neurons (Røe et al., 2000; Zhou, 2003). Consequently, the information received by the rested limbs is probably mediated through the bilateral distribution of the descending motor pathways (Røe et al., 2000; Zhou et al., 2002). The mechanisms underlying the contralateral effects of training are uncertain and may be caused by muscular, neural, spinal cord, cortical and subcortical influence (Kofotolis and Kellis, 2007). As no evidence of morphological muscle changes 
was reported in the contralateral limb (Zhou et al., 2002), the central neural mechanisms are believed to be mostly accountable for the cross education effects (Carroll et al., 2006; Gabriel et al., 2006; Kofotolis and Kellis, 2007; Lee et al., 2009; Munn et al., 2005; Zhou et al., 2002).

The terms co-agonists and co-antagonists were previously introduced to suggest the role of muscles in the non-exercised limb, based on their function in the exercised limb (Hellebrandt and Waterland, 1962). Thus an agonist muscle in the exercised limb is considered a co-agonist muscle in the contralateral-non-exercised-limb. Unilateral strength training appears to increase the strength in co-agonist muscles (Munn et al., 2005). The unilateral training produced an increase of $8 \%$ of initial strength of the co-agonist muscles (Munn et al., 2004). Some studies have examined the effect of unilateral training, however few addressed the cross education phenomenon related to PNF concept (Kofotolis and Kellis, 2007; Sato and Maruyama, 2009). Kofotolis and Kellis (2007) found increases of about $10 \%$ in the contralateral knee extension torque after 8 weeks of PNF unilateral training in the lower limbs. The aforementioned studies investigated the effect of cross education on isokinetic torque. However, most mechanisms underlying the effects of cross education remain unclear (Hendy et al., 2012). The assessment of muscle activity in the co-agonist and co-antagonist muscles during unilateral exercises might provide further insights on cross education phenomenon.

Pink (1981) suggested stabilization and central neural mechanisms as the two main phenomena underlying the activity of the contralateral non-exercised muscles. The stabilization mechanism would prevail when a muscle is similarly recruited when acting as either co-agonist or co-antagonist. The central neural mechanism, based on neurological overflow, would be mostly predominant when a muscle shows greater activity when acting as a co-agonist than co-antagonist. The author (Pink, 1981) evaluated the levels of activity in the non-exercised latissimus dorsi, infraspinatus and pectoralis major muscles of ten women while PNF patterns of diagonal for flexion and diagonal for extension were performed. Substantial levels of activation were observed in all muscles during both diagonal exercises. The supraspinatus and latissimus dorsi were more active when acting as co-agonists, supporting neural mechanisms as the main cause of this activity. The pectoralis major showed similar level of contraction between both diagonals and it is likely to be acting as a stabilizer (Pink, 1981). Further investigations assessing more subjects, including participants from both sexes, investigating isometric techniques, and exploring the activity of other shoulder muscles were suggested (Pink, 1981). To our knowledge, either the effects of force irradiation during isometric exercises based on PNF principles, or the profile of activity of co-agonist and co-antagonist muscles during these gestures remain unclear.

Higher intensity of training promotes greater magnitudes of cross education effects (Hendy et al., 2012). Indeed, the majority of studies applied contraction intensities of $60 \%$ of maximum voluntary contraction or greater (Hendy et al., 2012). However, the influence of different levels of effort on muscle activity on the non-exercised limb have not been addressed. Another issue barely explored is the effect of the sex of the participants on force irradiation. Differences on the level of muscle activity between men and women were already described (Pincivero et al., 2000). A better knowledge of the interaction between level of effort applied on exercises and sex on muscle activity via force irradiation might make clearer the role of different diagonal exercises in rehabilitation protocols.

The importance of exploring force irradiation phenomenon lies in the benefit of its clinical application in the presence of sensorimotor deficits caused by central or peripheral injuries, limb immobilization, pain, burns, fractures, as well as in preventing impairments related with muscle atrophy (Hazaki et al., 1996; Pink, 1981; Sato and Maruyama, 2009; Sullivan and Portney, 1980; Zhou, 2003). Therefore, the aim of this study was to explore the force irradiation effects of upper limb isometric diagonal exercises on shoulder muscle activities. Interactions among diagonal directions, contraction intensities (moderate and maximum) and sex were assessed.

\section{Methods}

\subsection{Participants}

This is a cross-sectional study with a convenience sample. Healthy university students were included in this research. Subjects with cognitive deficits, cardiac, pulmonary, musculoskeletal or neurologic diseases or presence of any pain were excluded. Nineteen women (with age of $20.95 \pm 1.17$ years old, weight of $57.00 \pm 6.05 \mathrm{~kg}$, and height of $163.00 \pm 5.36 \mathrm{~cm}$ ) and 11 men (with age of $20.95 \pm 2.12$ years old, weight of $68.94 \pm 6.44 \mathrm{~kg}$, and height of $176.11 \pm 6.94 \mathrm{~cm}$ ) were enrolled. The current investigation was approved by the local Ethic Committee and all the participants provided informed written consent based on the Declaration of Helsinki.

\subsection{Instruments}

Surface electromyography-sEMG (model MP100, Biopac System, California, USA) was used to acquire the myoelectric activity at a sample rate of $1000 \mathrm{~Hz}$. The pairs of active silver chloride $(\mathrm{AgCl})$ electrodes $150 \mathrm{~B}$ TDS circular surface with a diameter of $11.4 \mathrm{~mm}, 95 \mathrm{~dB} C \mathrm{CMRR}$ and input impedance of $100 \mathrm{M} \Omega$ were used. We used the Impedance Checker Noraxon ${ }^{\circledR}$ (Noraxon, Scottsdale, Arizona, USA) to measure the skin impedance. The isokinetic dynamometer Biodex System 4 Pro ${ }^{\circledR}$ (Biodex Medical Systems Inc., New York, USA) was used for both performing upper limb diagonal exercises and monitoring the muscle torque.

\subsection{Procedures}

Firstly, the upper limb dominance of the participants was verified by asking them what hand they use to write (Largo et al., 2001; Sullivan and Portney, 1980). Then, their height and weight were measured by a tape measure with a precision of $0.50 \mathrm{~cm}$ and a digital scale with a precision of $0.10 \mathrm{~g}$, respectively.

The sEMG evaluation was carried out on the non-dominant upper limb during isometric contractions performed in the isokinetic dynamometer by the dominant upper limb (Fig. 1). The participants performed two exercises with their dominant upper limbs: (i) isometric diagonal for flexion (hereafter referred to as flexion), which consists of isometric shoulder flexion, external rotation and abduction and (ii) isometric diagonal for extension (hereafter referred to as extension), which consists of isometric shoulder extension, internal rotation and adduction. The skin of the non-dominant upper limb was prepared as previously suggested: muscle mid-belly was shaved and the cell debris were removed. The proper skin cleansing procedure with $70 \%$ of ethyl alcohol was done. Then, the skin impedance was measured and it was considered acceptable when it was less than $5 \mathrm{k} \Omega$ (Basmajian and de Luca, 1985). For the sEMG analyses, bipolar configuration with $20 \mathrm{~mm}$ of inter-electrodes was adopted as criterion for distance. The electrodes were placed on the middle of the muscle belly, parallel to the direction of muscle fibers, and they were fixed with adhesive tape (SENIAM, n.d.). 


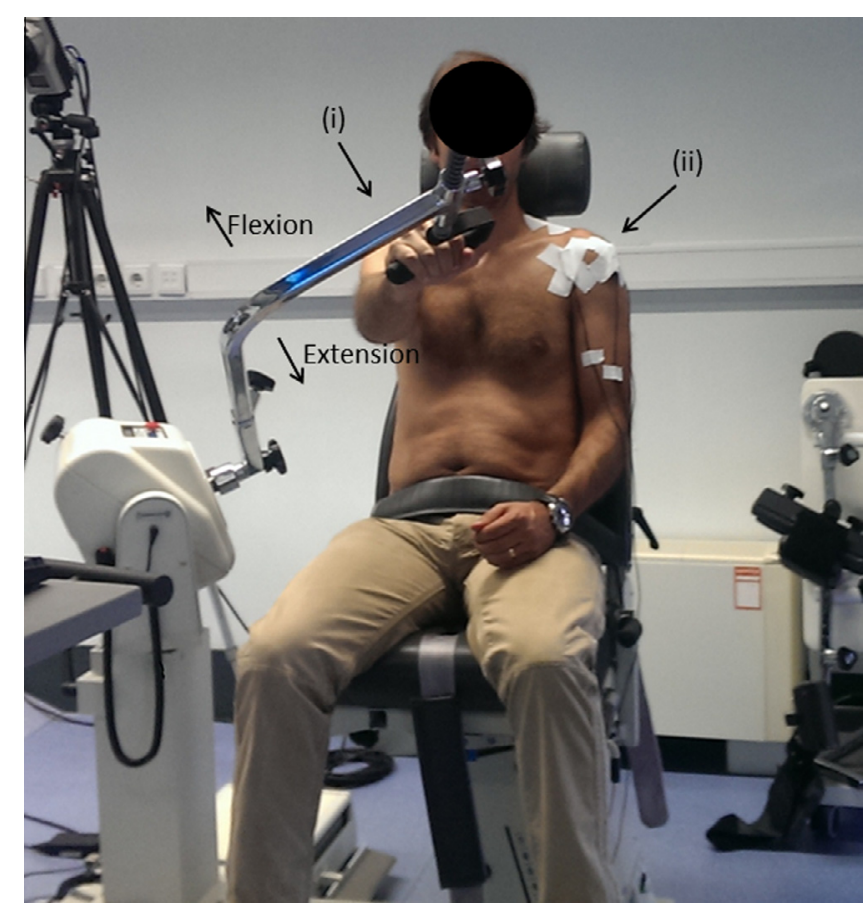

Fig. 1. Experimental setup. Participant positioned on the isokinetic dynamometer with his (i) dominant-exercised-limb and (ii) non-dominant-non-exercisedupper limb on test position. Flexion-diagonal for flexion (shoulder flexion, external rotation and abduction); Extension-diagonal for extension (shoulder extension, internal rotation and adduction).

The electrodes placement was based on the following references: (i) medial deltoid (MD): one finger from the distal and anterior face of the acromion with the orientation between acromion and thumb (Røe et al., 2000; SENIAM, n.d.); (ii) pectoralis major (PM): four fingers below the clavicle, medial to the anterior axillary border (Lehman et al., 2006); and (iii) upper trapezius (UT): at 50\% on the line from the acromion to the seven cervical vertebra (Røe et al., 2000; SENIAM, n.d.). The reference electrode was placed on the right fibular malleolus.

The participants were asked to briefly perform some shoulder elevations, abductions and horizontal abductions with their nondominant shoulders in order to test the electrodes EMG signal and crosstalk (Røe et al., 2000; SENIAM, n.d.). Then, the participants were instructed to perform a brief warm up with their non-dominant upper limbs. After that, they performed three maximum isometric voluntary contractions (MIVC) for each analyzed muscle against a manual resistance applied by the investigator (Lehman et al., 2006). These contractions were sustained for $5 \mathrm{~s}$. In order to reach the maximal rate of force development, the participants were asked to increase their force as fast as they could. The position and movement adopted to perform the MIVC were those suggested by previous studies (Fischer et al., 2011; Lehman et al., 2006-Table 1). The MIVC were used for normalization of the sEMG data.

Afterward, the participants were positioned at the isokinetic dynamometer in accordance with the "Diagonal Seated" protocol provided by the manufacture (Biodex System-Fig. 1). The seat orientation was $0^{\circ}$, while the dynamometer was orientated at $30^{\circ}$ and tilted $20^{\circ}$. The seat was positioned with a minimal height and adjusted to the anthropometric characteristics of the participants. Dynamometer height and the depth of the seat were adjusted to keep the dynamometer axis aligned with the participant's axilla. Then, the participant was stabilized with shoulder, waist and thigh straps. At the test position, the wrist in the dominant-exercised-upper limb was at neutral position, elbow was straight, and forearm pronated. The resistance offered by the dynamometer was on the participant's dominant hand. The shoulder of the non-dominant limb was in neutral position, the elbow was supported at about $120^{\circ}$ of flexion and the forearm and hand were at neutral position lying on the thigh (Fig. 1).

Following the preparation, the participants performed some active repetitions of both diagonals along their maximum range of motion. These active repetitions were used to establish the intermediate shoulder range of motion. The isometric exercises were performed at this intermediate position. This position was adopted as it is comfortable to the participants to perform both diagonals. Moreover, intermediate shoulder range of motion is widely used in clinical practice, for both orthopedic and neurological patients. It enhances the ecological validity of the present study. As the subjects included in the present study were healthy with no restrictions in range of motion, the intermediate position was very similar across all participants. During the trial, the participants remained seated with the assessed non-dominant upper limb "at rest" while the dominant upper limb performed the isometric contractions. The participants executed three trials of flexion and extension at a low intensity in order to familiarize with the trial. Both diagonal directions were performed with the participant's upper limbs at the same position.

The trials were carried out at two phases. During the first phase, the participants performed their maximum isometric torque (MIT). The trial started after an auditory feedback provided by the isokinetic dynamometer that instructed the participants to perform a flexion for $5 \mathrm{~s}$ followed by a rest period of $30 \mathrm{~s}$. Subsequently, a new auditory feedback instructed the participants to perform an extension for $5 \mathrm{~s}$ and then another $30 \mathrm{~s}$ rest. This procedure was performed until the participants completed three trials of flexion and three trials of extension. At the second phase, we used the values recorded in phase one to calculate $25 \%$ of the participants' MIT $\left(\mathrm{MIT}_{25 \%}\right.$ ) for both diagonals (average values of the three trials were used). Then, the participants performed six more diagonal exercises (three flexion and three extension), following the same instructions and position adopted in the first phase, but at $\mathrm{MIT}_{25 \%}$. This intensity was controlled by visual (dynamometer screen) and verbal (investigator information) feedbacks. Two minutes of rest between the phase one and two were granted to avoid fatigue (Devine et al., 1981; Hazaki et al., 1996). All procedures and verbal commands were executed by the same investigator for all participants.

\subsection{Data processing and analysis}

The data from the sEMG and isokinetic dynamometer were synchronized by an analog signal access interface (Biopac Systems Inc. California, USA). The EMG data were recorded from the beginning

Table 1

Description of positions and gestures used to the maximal isometric voluntary contractions.

\begin{tabular}{|c|c|}
\hline Muscle & Tests positions and gestures \\
\hline MD & $\begin{array}{l}\text { Seated with shoulder abducted } 90^{\circ} \text { in the plane of scapula, internally rotated (thumb down) and elbow extended. The arm was then abducted against a } \\
\text { resistance performed by the investigator (Fischer et al., 2011) }\end{array}$ \\
\hline PM & In supine with shoulder abducted to approximately 75 , was required the gesture of trying to take her hand inside (Lehman et al., 2006 ) \\
\hline UT & $\begin{array}{l}\text { Seated with the trunk against the chair back and their upper limbs along the trunk. The participant performed shoulder elevation by pulling upwards on both } \\
\text { handles simultaneously and without moving the shoulder girdle (Barbero et al., 2011) }\end{array}$ \\
\hline
\end{tabular}


(first variation of the torque curve) to the end of the contraction (rest).

EMG signal processing was completed using the Acqknowledge $^{\circledR} 3.9$ software (Biopac Systems Inc. California, USA). Signals were filtered with a high-pass filter of $50 \mathrm{~Hz}$ and a low-pass filter of $450 \mathrm{~Hz}$. Then, the Root Mean Square (RMS) amplitude with window width of $100 \mathrm{~ms}$ was calculated. The central $3 \mathrm{~s}$ from every trail was extracted for analyses. Then, the RMS amplitude average of the 3 repetitions of flexion and extension during MIT and $\mathrm{MIT}_{25 \%}$ for the MD, UT and PM were calculated. All the sEMG signals obtained were scaled by the MIVC (average of three repetitions) of the respective muscle. These values were presented as percentage of the MIVC multiplied by 100 (referred to as \%EMG $\mathrm{Eax}_{\text {) }}$ (Hazaki et al., 1996). Therefore, the primary outcome of this study was the \%EMG $\mathrm{E}_{\mathrm{Max}}$ of each muscle.

\subsection{Statistics}

Data normality was held by the Shapiro-Wilk test $(p>0.05)$. For descriptive statistics we used mean, standard error and $95 \%$ confidence interval. A repeated measures ANOVA with the diagonals (flexion and extension), contraction intensity (MIT and $\mathrm{MIT}_{25 \%}$ ), and muscles (MD, PM and UT) as within-subjects factors, and sex as between-subjects factor was used. Whenever any statistical significant difference was found, the Fisher's Least Significant Difference was calculated. The data were analyzed and outliers were removed. We only removed values over three standard deviations around the mean that, after analyzing EMG curve, we considered a fail in data acquisition; they accounted for less than $3 \%$ of the data. Statistical analysis was performed using the Statistica ${ }^{\circledR}$ v.8 (Statfoft $^{\circledR}$, Tulsa, USA) with an $\alpha$ value set at 0.05 .

\section{Results}

There were no statistically significant interactions among diagonal direction, contraction intensity, and sex in the muscle activity $(F(2,293)=1.9710, p=0.14)$. However, statistically significant interactions were observed between diagonal direction and contraction intensity $(F(2,293)=4.2770, p=0.0147)$. In both diagonals, all muscles showed greater activity during MIT compared to $\operatorname{MIT}_{25 \%}$ ( $p<0.01-$ Fig. 2). The highest differences occurred in the
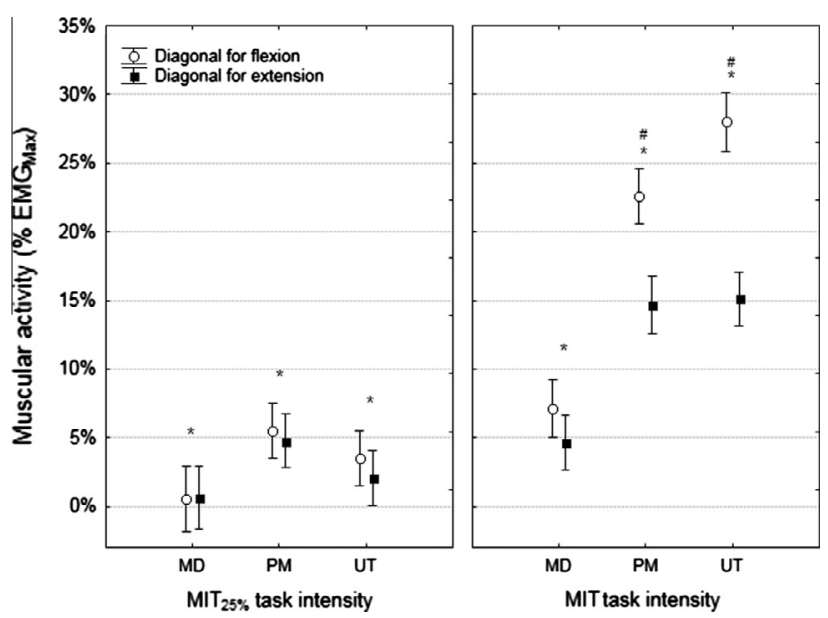

Fig. 2. Interactions between contraction intensity and diagonal directions in muscle activity (mean and 95\% confidence interval). MIT-maximum isometric torque; $\mathrm{MIT}_{25 \%}-25 \%$ of the maximum isometric torque; \% $\mathrm{EMG}_{\mathrm{Max}}$ - percentage of maximum isometric voluntary contraction; MD-medial deltoid; PM-pectoralis major; UP-upper trapezius; "-differences between contraction intensities ( MIT $_{25 \%}$ vs. MIT) with $p<0.05$; \#-differences between diagonal directions (flexion vs. extension) with $p<0.05$.
UT when during the $\mathrm{MIT}_{25 \%}$ showed values of $3.4 \pm 0.4 \% \mathrm{EMG}_{\mathrm{Max}}$ and $2.0 \pm 0.2 \% \mathrm{EMG}_{\mathrm{Max}}$ for flexion and extension, respectively; whilst during the MIT, the UT reached values of $27.6 \pm 1.4 \% \mathrm{EMG}_{\mathrm{Max}}$ and $14.2 \pm 2.0 \% \mathrm{EMG}_{\mathrm{Max}}$ for flexion and extension, respectively (Fig. 2).

Considering diagonal direction (flexion vs. extension), no statistically significant differences were found in the MD during either MIT or $\operatorname{MIT}_{25 \%}(p>0.05)$. For the PM, we also observed similar values $(p>0.05)$ between diagonals during the $\mathrm{MIT}_{25 \%}$. However, during the MIT the PM showed greater activity $(p<0.001)$ during the flexion $\left(22.3 \pm 1.2 \% \mathrm{EMG}_{\mathrm{Max}}\right)$ compared to the extension $\left(15.2 \pm 1.3 \% \mathrm{EMG}_{\mathrm{Max}}\right)$. This behavior was also observed in the UT: no statistically significant differences between diagonals at $\mathrm{MIT}_{25 \%}(p=0.33)$, and higher values during MIT for the flexion compared to the extension ( $p<0.001-$ Fig. 2$)$.

There were also statistically significant interactions between sex and contraction intensity $(F(1,293)=4.5146, p=0.03)$. Similar values were found between men $\left(2.9 \pm 0.3 \% \mathrm{EMG}_{\mathrm{Max}}\right)$ and women

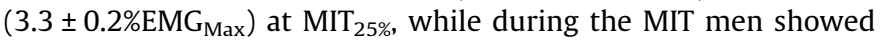
statistically significant higher muscle activity compared to women $\left(16.3 \pm 1.4\right.$ vs. $13.9 \pm 1.0 \% \mathrm{EMG}_{\mathrm{Max}}-$ Fig. 3$)$.

We also observed main effects of sex in the muscle activity $(F(2,293)=3.6012, p=0.028)$. Similar values between men and

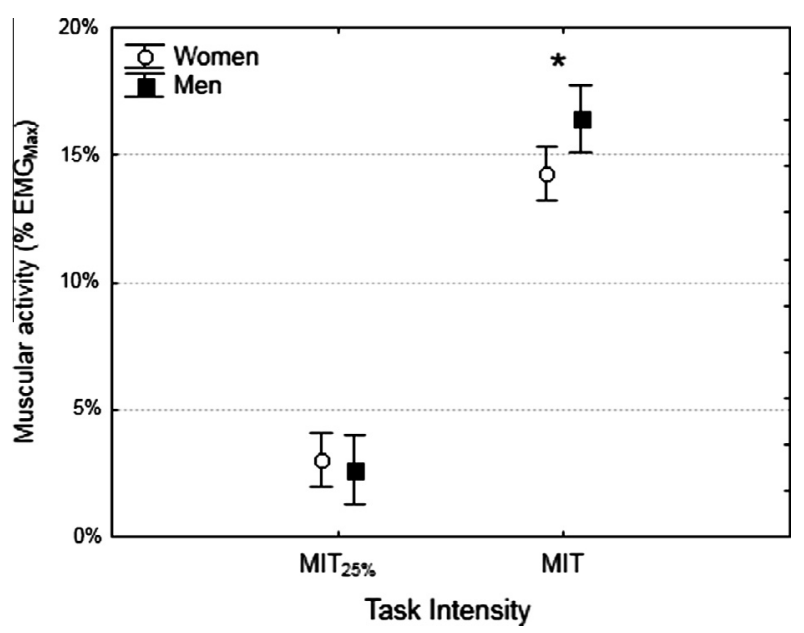

Fig. 3. Interactions between sex and contraction intensity in muscle activity (mean and $95 \%$ confidence interval). MIT-maximum isometric torque; $\mathrm{MIT}_{25 \%}-25 \%$ of the maximum isometric torque; \% $\mathrm{EMG}_{\mathrm{Max}}$-percentage of maximum isometric voluntary contraction; ${ }^{*} p<0.05$ differences between men and women.

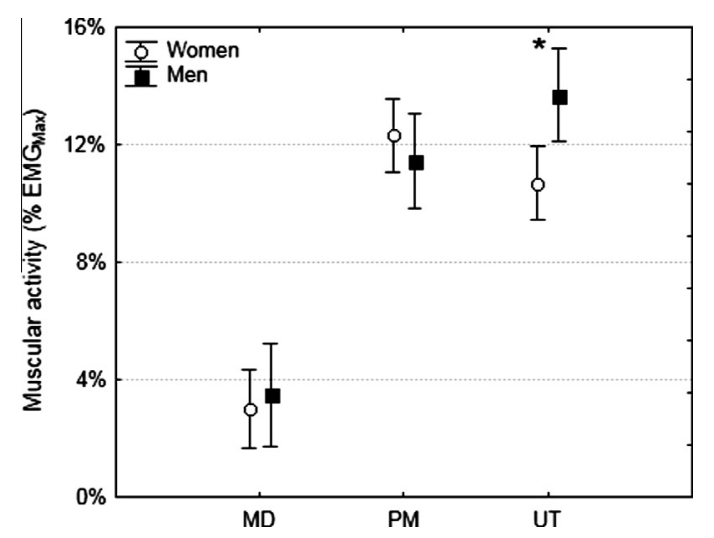

Fig. 4. Main effects of sex in muscle activity (mean and 95\% confidence interval). \% $\mathrm{EMG}_{\mathrm{Max}}$-percentage of maximum isometric voluntary contraction; MD-medial deltoid; PM-pectoralis major; UP-upper trapezius; " $p<0.05$ differences between men and women. 
women were found in the MD and PM, however statistically significant greater values were observed in the UT for men compared to women (Fig. 4).

\section{Discussion}

In the present study we observed increases in muscle activity in all studied muscles during the highest contraction intensity (MIT) compared to $\mathrm{MIT}_{25 \%}$. Furthermore, the diagonal direction also influenced muscle activities: the PM and UT showed higher values during the flexion compared to the extension. Finally, we observed significant interactions between contraction intensity and sex, and main effects of sex on the muscle activity.

We observed increases between 1.7 and $27 \% \mathrm{EMG}_{\mathrm{Max}}$ in the muscle activity at the non-exercised upper limb for the PM, UT and MD during diagonal exercises. These muscles were selected in the current study as they present important actions to either the flexion or extension (Vandenberghe et al., 2012). Therefore, the design of the present study allowed exploring the role of coagonists and co-antagonists during diagonal exercises. In a previous study, Pink (1981) assessed isotonic contractions with manual resistance while the participants were in supine position. Increases between 3 and $7 \% \mathrm{EMG}_{\mathrm{Max}}$ for the latissimus dorsi, PM and infraspinatus in the non-exercised upper limb during flexion and extension were observed (Pink, 1981). The author did not find greater muscle activity of the PM when it acted as a co-agonist. The PM presents an important action in the shoulder adduction and internal rotation-components of the diagonal for extension. We observed similar values between diagonal directions during the $\mathrm{MIT}_{25 \%}$ task, and lower levels of activation during flexion. Our findings corroborate those of Pink (1981) and suggest the PM is actively contracting in order to stabilize the movement. The similar levels of activation in the MD between diagonals suggest this muscle has stabilizing functions as well.

Røe et al. (2000) found increased muscle activity in the UT from the non-exercised upper limb during resisted shoulder abduction. We observed higher values for the UT during the diagonal for flexion, where there is a component of shoulder abduction, compared to extension. These findings could result from the fact that trapezius muscles have a bilateral cortical connection and crossed mono-synaptic reflexes. The UT from the non-exercised side is likely to be more active as it has common pre-synaptic motor neurons and helps to maintain the spine position during arm elevation (Alexander et al., 2007). Our findings and that of the aforementioned study (Røe et al., 2000) support greater muscle activity for the UT when it acts as a co-agonist. As a result the central mechanisms of neurologically based overflow are supported.

The PNF technique highlights the application of maximum resistance (Adler et al., 2000). In the current study, the muscle activity was analyzed during both maximum and partial levels of contractions (MIT and $\mathrm{MIT}_{25 \%}$ ). Our data suggest only low muscle activities occurred in the non-exercised upper limb during partial contractions (between 1.7 and 6\%EMG $\mathrm{Max}_{\mathrm{M}}$ ). During maximum effort, levels of muscle activity ranged from 5 to $27 \% \mathrm{EMG}_{\mathrm{Max}}$. One study (Fimland et al., 2009) assessed the effects of unilateral training in the non-exercised side and observed insignificant cross education effects on the plantar flexors after 4 weeks of isometric training at $32 \% \mathrm{EMG}_{\mathrm{Max}}$. It is believed that only training with intensities equal or higher than $50 \% \mathrm{EMG}_{\mathrm{Max}}$ lead to cross education effects with increase of strength and muscle activity (Sato and Maruyama, 2009; Zhou et al., 2002). Our data supports that a task performed at $25 \%$ of the MIT provided increases in muscle activity in the non-exercised shoulder muscles lower than $6 \% \mathrm{EMG}_{\mathrm{Max}}$. Therefore, when the aim is to maximize the muscle activity in the non-exercised upper limb, our data provide some evidence suggesting that flexion at MIT is the most adequate gestures to be applied.

Differences between sexes were found in the present study. Our findings support similar levels of muscle activity between men and women for the MD and PM, and greater muscle activity for the UT in men compared to women. Previous studies described differences in the patterns of antagonists and stabilizers between sexes (Adamo et al., 2012; Pincivero et al., 2000). Pincivero et al. (2000) observed higher RMS amplitude in men compared to women. Data from the present study did not allow speculation on the mechanisms underlying this sex differences.

The current study presents some limitations. We were not able to accomplish some principles of the PNF method, such as hand position and manual contact. Such measures were needed in order to allow a standardized protocol. However, most PNF principles, such as diagonal movement, verbal incentive and visual stimuli were included. We did not verify any post-trial effect. In the present study only healthy participants were assessed. It is unclear whether or not our findings reflect the behavior of populations with neurologic or orthopedic impairments. Future studies assessing cross education effects on population of patients, and making clearer the clinical relevance of considering sex and level of effort when selecting the exercises are needed.

\section{Conclusion}

Unilateral diagonal exercises based on PNF method increased the muscle activity in the non-exercised shoulder muscles. The force irradiation effects were higher during a maximum effort (MIT) compared to the partial effort task ( $\mathrm{MIT}_{25 \%}$ ). The diagonal direction also influenced the muscle activity. The flexion showed higher muscle activity in the UT and PM than the extension. Furthermore, men and women showed different muscle activity levels during the MIT task. Overall, contraction intensity, diagonal direction and sex might be relevant factors to be considered in rehabilitation protocols when the aim is to use the force irradiation principle to activate shoulder muscles in the non-exercised upper limb. Our data suggests that force irradiation may be effective to improve muscle activity in situations in which the upper limb cannot be actively exercised. 


\section{References}

Adler S, Beckers D, Buck M. PNF in practice - an illustrated guide. New York: Springer-Verlag; 2000.

Adamo DE, Scotland S, Martin BJ. Upper limb kinesthetic asymmetries: gender and handedness effects. Neurosci Lett 2012;516:188-92.

Alexander C, Miley R, Stynes S, Harrison PJ. Differential control of the scapulothoracic muscles in humans. J Physiol 2007;580:777-86.

Barbero M, Gatti R, Conte LL, Macmillan F, Coutts F, Merletti R. Reliability of surface EMG matrix in locating the innervation zone of upper trapezius muscle. J Electromyogr Kinesiol 2011;21:827-33.

Basmajian JV, de Luca C. Muscles alive, their function revealed by electromyography. 5th ed. Maryland: Williams and Wilkins: 1985.

Biodex. Biodex Multi-Joint System-Pro. New York: Biodex Medical System Inc.

Carroll TJ, Herbert RD, Munn J, Lee M, Gandevia SC. Contralateral effects of unilateral strength training: evidence and possible mechanisms. J Appl Physiol 2006; 101:1514-22.

Devine KL, LeVeau BF, Yack HJ. Electromyographic activity recorded from an unexercised muscle during maximum isometric exercise of the contralateral agonists and antagonists. Phys Ther 1981;61:898-903.

Dimitrijevic MR, McKay WB, Sarjanovic I, Sherwood AM, Svirtlih L, Vrbova G. Coactivation of ipsi- and contralateral muscle groups during contraction of ankle dorsiflexors. J Neurol Sci 1992;109:49-55.

Fimland MS, Helgerud J, Solstad GM, Iversen VM, Leivseth G, Hoff J. Neural adaptations underlying cross-education after unilateral strength training. Eur J App Physiol 2009;107:723-30.

Fischer SL, Grewal TJ, Wells R, Dickerson CR. Effect of bilateral versus unilateral exertion tests on maximum voluntary activity and within-participant reproducibility in the shoulder. J Electromyogr Kinesiol 2011;21:311-7.

Gabriel DA, Kamen G, Frost G. Neural adaptations to resistive exercise: mechanisms and recommendations for training practices. Sports Med 2006;36:133-49.

Gontijo LB, Pereira PD, Neves CDC, Santos AP, Machado DCD, Bastos VHV. Evaluation of strength and irradiated movement pattern resulting from trunk motions of the proprioceptive neuromuscular facilitation. Rehabil Res Pract 2012:1-6.

Hazaki K, Ichihashi N, Morinaga T. Electromyographic analysis of thigh muscles in PNF patterns of the lower extremity: muscle activities in the lengthened range. J Phys Ther Sci 1996;8:29-32.

Hellebrandt FA, Waterland JC. Indirect learning. The influence of unimanual exercise on related muscle groups of the same and the opposite side. Am J Phys Med 1962;41:45-55.

Hendy AM, Spittle M, Kidgell DJ. Cross education and immobilisation: mechanisms and implications for injury rehabilitation. J Sci Med Sport 2012;15:94-101.

Kofotolis ND, Kellis E. Cross-training effects of a proprioceptive neuromuscular facilitation exercise programme on knee musculature. Phys Ther Sport 2007;8:109-16.

Largo RH, Caflisch JA, Hug F, Muggli K, Molnar AA, Molinari L, et al. Neuromotor development from 5 to 18 years. Part 1: timed performance. Dev Med Child Neurol 2001;43:436-43.

Lee LJ, Coppieters MW, Hodges PW. Anticipatory postural adjustments to arm movement reveal complex control of paraspinal muscles in the thorax. J Electromyogr Kinesiol 2009;19:46-54.

Lehman GJ, MacMillan B, MacIntyre I, Chivers M, Fluter M. Shoulder muscle EMG activity during push up variations on and off a Swiss ball. Dyn Med 2006;9(5):7.

Meningroni PC, Nakada CS, Hata L, Fuzaro AC, Marques W, Araujo JE, et al. Contralateral force irradiation for the activation of tibialis anterior muscle in carriers of Charcot-Marie-Tooth disease: effect of PNF intervention program. Braz J Phys Ther 2009; 13:438-43.

Moore JC. Excitation overflow: an electromyographic investigation. Arch Phys Med Rehabil 1975;56:115-20.

Munn J, Herbert RD, Gandevia SC. Contralateral effects of unilateral resistance training: a meta-analysis. J Appl Physiol 2004;96:1861-6.

Munn J, Herbert RD, Hancock MJ, Gandevia SC. Training with unilateral resistance exercise increases contralateral strength. J Appl Physiol 2005;99:1880-4.

Panzer S, Schinowski1 D, Kohle D. Cross-education and contralateral irradiation. J Hum Kinet 2011:27:66-79.

Pincivero DM, Green RC, Mark JD, Campy RM. Gender and muscle differences in
EMG amplitude and median frequency, and variability during maximal voluntary contractions of the quadriceps femoris. J Electromyogr Kinesiol 2000;10:189-96.

Pink M. Contralateral effects of upper extremity proprioceptive neuromuscular facilitation patterns. Phys Ther 1981;61:1158-62.

Røe C, Brox JL, Saugen E, Vøllestad NK. Muscle activation in the contralateral passive shoulder during isometric shoulder abduction in patients with unilateral shoulder pain. J Electromyogr Kinesiol 2000;10:69-77.

Sato H, Maruyama H. The effects of indirect treatment of proprioceptive neuromuscular facilitation. J Phys Ther Sci 2009;21:189-93.

SENIAM. Surface ElectroMyoGraphy for the non-invasive assessment of muscles. Retrieved at 23rd of November 2011; n.d.

Sullivan PE, Portney LG. Electromyographic activity of shoulder muscles during unilateral upper extremity proprioceptive neuromuscular facilitation patterns. Phys Ther 1980;60:283-8.

Vandenberghe A, Bosmans, De Schutter J, Swinnen S, Jonkers I. Quantifying individual muscle contribution to three-dimensional reaching tasks. Gait Posture 2012;35:579-84.

Voss DE, Ionta MK, Myers BJ. Proprioceptive neuromuscular facilitation. 3rd ed. Philadelphia, PA: Harper \& Row; 1985.

Zhou SA. Cross education and neuromuscular adaptations during early stage of strength training. J Exerc Sci Fit 2003;1:54-60.

Zhou S, Oakman A, Davie AJ. Effects of unilateral voluntary and electromyostimulation training on muscular strength on the contralateral limb. J Exerc Sci Fit 2002;XIV:1-11. 